\title{
DEUX GROUPES DE COLLEGIENS (ABIDJAN ET TOULOUSE) FACE A DEUX QUESTIONS SUR LA TRANSITIVITE ET L'INTRANSITIVITE EN FRANÇAIS: RECHERCHES SUR LES CONNAISSANCES IMPLICITES ET EXPLICITES DES ADOLESCENTS
}

\author{
CHIARA DE ANGELIS, ORESTE FLOQUET ${ }^{1}$
}

\begin{abstract}
Two groups of high school students (of Abidjan and Toulouse) answering two questions on transitivity and intransitivity in the French language: a research on teenagers' implicit and explicit knowledge. By employing the epilinguistic and metalinguistic analysis of a corpus of responses by 170 pre-teenagers from two colleges in Abidjan and Toulouse, we will discuss, first, the concepts of acceptability, grammaticalness and utterability in order to try to determine, as far as possible, on what kind of knowledge and on what type of reasoning they rely on. Secondly, we will discuss the relationship that connects acceptability and age by showing that, contrary to what is expected, the majority of pre-teenagers that we have questioned still ground their responses more in their encyclopedic knowlege than in the formal aspects of the phrase, which is a typical feature of adolescence. ${ }^{2}$
\end{abstract}

Keywords: acceptability, valency, metalinguistic awareness, language development.

REZUMAT. Cum răspund două grupuri de liceeni (din Abidjan și Toulouse) la două întrebări despre tranzitivitate și intranzitivitate în limba franceză: o cercetare asupra cunoștințelor implicite și explicite ale adolescenților. Prin intermediul unei analize epilingvistice și metalingvistice a unui ansamblu de răspunsuri preluate de la 170 de preadolescenți provenind de la două licee din Abidjan și, respectiv, Toulouse, vom discuta, mai întâi, noțiunile de acceptabilitate, gramaticalitate și enunțiabilitate, în încercarea de a stabili, pe cât se poate, care sunt cunoștințele și tipurile de raționamente pe care se bazează acestea. Apoi, vom analiza raportul care se stabilește între acceptabilitate și vârstă, arătând că, contrar așteptărilor noastre, majoritatea preadolescenților chestionați își

\footnotetext{
${ }^{1}$ Chiara DE ANGELIS, Sapienza, Università di Roma, est responsable du codage des protocoles. Oreste FLOQUET, Sapienza, Università di Roma, est responsable du codage des protocoles et de l'écriture de l'article. Un vif remerciement à Maria Antonietta Pinto, Chiara Celata et Giulia De Flaviis qui ont eu la gentillesse de relire la première version de cet article. De toute évidence, elles ne sont en aucun cas responsables des imperfections subsistantes.

E-mail : chiara.de1996@gmail.com

E-mail : oreste.floquet@uniroma1.it

2 The abstract has been translated into English and Romanian by Ioana-Gabriela Nan.
} 


\begin{abstract}
argumentează încă răspunsurile referindu-se mai degrabă la cunoștințele lor enciclopedice decât la aspectele formale ale frazei, ceea ce reprezintă o trăsătură tipică vârstei adolescente.
\end{abstract}

Cuvinte cheie: acceptabilitate, valență, conștiință metalingvistică, dezvoltare lingvistică.

\title{
1. Introduction
}

C'est un fait notoire que les locuteurs natifs aussi bien que les apprenants d'une L2 ont parfois des intuitions, des représentations et des connaissances stockées en mémoire qui leur sont propres et qui peuvent s'éloigner de la norme prescriptive (SORACE 1985; TREVISE 1994; BEACCO 2001; WEBER 2004; DEBRENNE 2007). La question que nous nous posons dans cet article, qui n'est pas d'ordre pédagogique ou didactique (COLOMB01982; WEBER 2008; NADEAU et FISHER 2011), est de savoir quelle place accorder, dans la recherche, aux connaissances grammaticales, implicite et explicite, des locuteurs natifs dans la phase de la pré-adolescence. Plus précisément, l'observation des appréciations spontanées et des commentaires métalinguistiques que nous avons menée sur deux groupes de collégiens à propos de deux constructions non canoniques (transitive et intransitive) vont nous amener à des considérations plus générales concernant l'âge par rapport au sentiment épilinguistique superficiel, qui est une compétence intuitive et opérative des règles (le « grammatical feeling » ou « Sprachgefühl »; TITONE 1983: 242) et les représentations métalinguistiques que l'on peut verbaliser et contrôler de manière intentionnelle et qui sont le fruit aussi bien de l'apprentissage scolaire que d'un raisonnement individuel plus autonome. À partir de quel âge un locuteur à qui on demande une appréciation sur une phrase incorrecte est capable de s'apercevoir des fautes éventuelles et surtout de distinguer celles qui relèvent de la syntaxe de celles qui concernent la sémantique et la pragmatique? Y a-t-il une différence entre le comportement des enfants et celui des pré-adolescents? Le but de cet article est d'approfondir ces questions ainsi que leurs retombées théoriques à partir d'un corpus d'intuitions d'acceptabilité de collégiens de deux différentes aires de la francophonie qui a été élicité en suivant le protocole prévu dans PINTO et EL EUCH (2015) pour cette tranche d'âge.

\section{L'acceptabilité}

Par acceptabilité nous entendons une habileté que possède tout un chacun et qu'à la suite de CRYSTAL (2008: 4) nous pouvons définir comme: 
DEUX GROUPES DE COLLÉGIENS (ABIDJAN ET TOULOUSE) FACE À DEUX QUESTIONS SUR LA TRANSITIVITÉ ...

« the extent to which linguistic data would be judged by native-speakers to be possible in their language. An acceptable utterance is one whose use would be considered permissible or normal ».

Premièrement, une telle capacité est double, car il faut soigneusement distinguer ces jugements qui sont effectués spontanément et dont la nature est plutôt binaire, (p.e.: « c'est correct » ou bien « ce n'est pas acceptable ») de ceux qui sont le fruit d'une réflexion explicite pouvant donner lieu à une argumentation plus ou moins développée (p.e. "c'est correct parce que ...», «ce n'est pas acceptable car ... »). Il faut donc séparer ce qui relève de la « conscience » de ce qui manifeste plutôt une "prise de conscience » (BREDART et RONDAL 1982: 16). Dans la suite de cet article, nous allons parler d'activité épilinguistique lorsque le jugement d'acceptabilité est produit incosciemment et d'activité métalinguistique si le jugement est, en revanche, le produit d'une délibération consciente (GOMBERT 1990: 22; LA MANTIA 2017).

Deuxièmement, l'acceptabilité n'est jamais générale mais elle se décline suivant les différents niveaux de la langue. Pour rester aux aspects qui nous intéressent ici, nous allons distinguer la grammaticalité syntaxique qui consiste à savoir évaluer la forme d'une phrase de la plausibilité sémantique qui concerne tantôt la compatibilité des différents contenus lexicaux entre eux, tantôt leur correspondance avec le monde extérieur auquel ils se réfèrent. Dans les deux cas, pour pouvoir répondre à une question concernant l'acceptabilité d'une phrase, il faut au préalable avoir intériorisé un modèle (qu'il soit conforme à la norme officielle ou pas, peu importe) pour pouvoir ensuite effectuer la comparaison.

Troisièmement, l'acceptabilité est un concept plus vaste que celui de grammaticalité, car, dans la littérature psycholinguistique et linguistique (p.e. PETERFALVI et LOCATELLI 1971, DESCLÉS et GUENTCHÉVA 1991), il se réfère aussi bien aux jugements concernant la violation (ou pas) des agencements formels de la phrase que les considérations sur l'interprétation d'un énonçé en fonction de différents paramètres (p.e., le co-texte, la situation, la vitesse du débit ou la longueur d'une phrase, etc.).

Telle qu'elle est conçue par PINTO et EL EUCH (2015), l'épreuve d'acceptabilité du THAM-2 permet en effet de tenir compte simultanément de ces trois aspects: (1) les questions portent aussi bien sur le niveau intuitif (p.e. " peut-on dire ça? ») que sur un niveau plus riche, qui est celui de l'argumentation (p.e. « peux-tu expliquer ta réponse? »); (2) le sujet est appelé à juger une phrase présentant soit une anomalie sémantique soit une anomalie grammaticale; le test ne vise par conséquent que certains niveaux précis de la langue; (3) grâce à la partie argumentative de la réponse, le chercheur peut essayer de comprendre si la réponse épilinguistique se fonde sur un sentiment d'agrammaticalité au sens strict du terme (et porte donc sur le statut formel de la phrase), si l'enquêté prend plutôt en considération des facteurs propres à une situation donnée (et 
s'intéresse davantage au caractère plus ou moins énonçable, plus au moins plausible, d'un séquence) ou bien si l'accent est mis sur des aspects non prévisibles. Par ailleurs, il est possible de voir si le sujet répond en se basant sur des connaissances qui renvoient à la norme scolaire, à un usage particulier ou bien si elles sont le produit d'un raisonnement autonome. De toute évidence, comme le rappelle à juste titre WEBER (2008: 219): «il convient d'être prudent face aux nuances probables entre ce qu'ils [les sujets enquêtés] disent, ce qui est fait et ce qu'ils savent ».

\section{L'acceptabilité et l'âge}

De nombreuses études ont déjà montré combien il est difficile d'obtenir des jugements d'acceptabilité chez les enfants en bas âge bien que ceux-ci sachent utiliser correctement la syntaxe et la sémantique de leur langue dans leurs productions spontanées (TUNMER et GRIEVE 1984). On peut penser qu'il s'agit d'une habileté que les enfants doivent encore développer ou bien plus banalement que la méthode pour éliciter leurs jugements n'est pas suffisamment claire, qu'ils ne comprennent pas la question qu'on leur pose tout en ayant la capacité d'y répondre ${ }^{3}$. Chose importante pour nos fins, on sait que les facteurs sémantiques jouent un rôle plus important que les facteurs syntaxiques dans les jugements d'acceptabilité des enfants (DE VILLIERS et DE VILLIERS 1972). S'ils peuvent donner un sens à une phrase, les enfants l'accepteront, quelle que soit sa forme (p.e. avec un ordre de mots inverti ou avec des morphes effacés, etc.), ce qui signifie que pour eux, dans la phase de décodage, la syntaxe n'est pas encore une source d'information. Les enfants considèrent ainsi recevables des phrases qui sont rejetées par les adultes montrant un plus faible degré de discrimination alors qu'ils sont beaucoup plus sensibles aux anomalies de contenu. On en déduit que leur compétence sémantique semble se développer bien avant leur compétence syntaxique et qu'ils sont plus axés sur l'expérience concrète plutôt que sur les aspects de la structure linguistique. D'où la question: à quel age se stabilise un comportement « adulte »? Les études psycholinguistiques menées sur ce sujet (généralement sur des populations anglophones) indiquent que ce n'est que vers l'âge de 8 ans que les enfants deviennent enfin capables de séparer le contenu d'une phrase de sa forme et d'identifier les phrases comme acceptables ou non uniquement pour des raisons linguistiques (s'entend formelles). Les résulats de notre enquête vont problématiser l'idée d'un seuil au-delà duquel la compétence linguistique adulte sera acquise une fois pour toutes en montrant des "résurgences» d'un comportement apparemement plus « archaïque » encore entre 11 et 14 ans.

${ }^{3}$ Comme le rappelle GOMBERT (1990: 23): « la non-explicitation ne suppose pas obligatoirement la non-conscience». 
DEUX GROUPES DE COLLÉGIENS (ABIDJAN ET TOULOUSE) FACE À DEUX QUESTIONS SUR LA TRANSITIVITÉ ...

\section{Le corpus et la passation}

Notre corpus se compose des réponses de 58 collégiens de 6ème, 59 de 5 ème et 53 de 4ème pour un total de 170 adolescents provenant de deux collèges d'Abidjan et de Toulouse, dont 110 étudiants ivoiriens et 60 français. À Toulouse, la passation a eu lieu en 2017; elle a été effectuée sous la direction de Maria Antonietta Pinto ${ }^{4}$. À Abidjan, elle s'est déroulée en deux phases, toujours dans le même collège, d'abord en 2018 (sous la direction de Béatrice Akissi Boutin) puis en 2019 (sous la direction d'Oreste Floquet). La totalité des collégiens est de langue maternelle française. Les enquêteurs leur ont explicitement demandé de s'exprimer sur les langues parlées en famille en les différenciant de celles qui ont été apprises à l'école. Seuls 28 étudiants ont déclaré parler une deuxième, voire une troisième langue: la plupart d'entre eux prétendent parler l'anglais, le reste le baoulé, le dioula, le beti ou l'espagnol.

Le protocole d'administration suivi ainsi que les consignes fournies aux étudiants ont été toujours les mêmes. Comme le manuel du THAM-2 le prévoit, il s'est agi d'abord de familiariser les collégiens à l'épreuve en leur proposant un premier item qui a fait l'objet d'un commentaire collectif de toute la classe, puis chaque étudiant a continué en répondant aux questions de manière individuelle. Il a été répété plusieurs fois que le test n'allait évaluer ni la compétence grammaticale ni la rapidité et qu'il n'y avait pas de bonne ou de mauvaise réponse, l'intérêt de la recherche étant plutôt de faire émerger des «points de vue » différents, tous légitimes au même degré.

Dans la suite de cet article, nous allons nous concentrer uniquement sur les résulats de ces quatre items qui sont les seuls qui, dans le THAM-2, présentent des anomalies concernant les constructions transitives et intransitives (dans la partie B de chaque paire):

3.2.A « la fille caressait le chien »

3.2.B « la fille caressait »

3.3.A « le papa éternuait»

3.3.B « la papa éternuait la voiture »

Pour chaque item, comme nous l'avons déjà dit, le test prévoit une question du type «peut-on dire cela? » puis une question du type «peux-tu expliquer ta réponse?». Nous allons commenter plus en détail les réactions épilinguistiques et les commentaires métalinguistiques de 3.2.B et 3.3.B.

\footnotetext{
${ }^{4}$ Auteure de la source originale du test THAM-2, d'abord conçu en langue italienne (PINTO,
} 1999), ensuite co-auteure de la traduction-adaptation du test en langue française. 


\section{Les réponses épilinguistiques globales et l'item « la fille caressait »}

À l'exception de 3.2.B, qui fera l'objet d'un commentaire spécifique, les intuitions épilinguistiques des élèvesne sont pas particulièrement surprenantes, les rares cas de réponses incertaines (p.e. « oui et non») ou déviantes (p.e. la réponse «oui » à «la fille éternuait la voiture ») n'étant pas suffisamment consistantes d'un point de vue quantitatif, sont probablement à ranger soit du côté d'une inattention momentantée soit d'un excès de prudence. Nous verrons toutefois dans le paragraphe 6 que les justifications sont, en revanche, loin d'être anodines.

Table 1. pourcentage global des réponses épilinguistiques (6ème, 5 ème et 4ème, Abidjan et Toulouse).

\begin{tabular}{|l|l|c|c|c|}
\hline \multicolumn{1}{|c|}{ item } & \%oui & \%non & \%incertaines \\
\hline 3.2.A & «la fille caressait le chien » & 99,4 & - & 0,6 \\
\hline 3.2.B & «la fille caressait » & 33 & 58 & 9 \\
\hline 3.3.A & «le papa éternuait » & 97,6 & 1,2 & 1,2 \\
\hline 3.3.B & « la papa éternuait la voiture » & 0,6 & 98,2 & 1,2 \\
\hline
\end{tabular}

Pour ce qui est des deux items $B$, où se nichent des anomalies de sélection («caresser» nécessitant un argument interne contrairement à « éternuer » dont la construction est intransitive), les tendances qui émergent du tableau 1 sont confirmées tantsi on prenden considération les différents niveaux scolaires que si on analyse les variables diatopiques:

Table 2. Réponses épilinguistiques positives et négatives suivant les trois classes concernant 3.2.B e 3.3.B.

\begin{tabular}{|c|c|c|c|c|c|}
\hline & item & classe & $\%$ oui & $\%$ non & $\%$ incertaines \\
\hline 3.2.B & & 6ème & 34 & 59 & 7 \\
\hline & « la fille caressait » & 5ème & 30,5 & 61 & 8,5 \\
\hline & & 4ème & 34 & 53 & 13 \\
\hline 3.3.B & & 6ème & - & 98,3 & 1,7 \\
\hline & « le papa éternuait la voiture» & 5ème & - & 98,3 & 1,7 \\
\hline & & 4ème & - & 98,1 & 1,9 \\
\hline
\end{tabular}

Table 3. Réponses épilinguistiques concernant les items 3.2.B e 3.3.B réparties suivant les classes et les lieux d'enquête.

\begin{tabular}{|l|l|c|c|c|}
\hline item & ville & \% oui & \% non & \% incertaines \\
\hline 3.2.B & Abidjan & 40 & 53 & 7 \\
\cline { 3 - 5 } 3.3.B & & - & 99,1 & 0,9 \\
\hline 3.2.B & \multirow{2}{*}{ Toulouse } & 27 & 66 & 7 \\
\hline $3.3 . \mathrm{B}$ & & 1,7 & 96,6 & 1,7 \\
\hline
\end{tabular}


À moins de ne considérer extrinsèque toute la procédure d'élicitation des données de l'épreuve, argument légitime mais qui, soit dit en passant, n'est pas forcément résolu ni par la multiplication des inputs proposés (car les contextes et les contraintes sont nombreux) ni par le dépouillement d'un grand corpus de formes attestées (car l'utilisation d'une forme par un locuteur ne nous dit riensur la valeur que ce dernier lui attribue par rapport à d'autres formes qui lui seraient plus ou moins concurrentes, plus ou moins acceptables, plus ou moins synonymes, etc.), le flottement dans les intuitions concernant l'acceptabilité de «la fille caressait» mérite, à notre avis, un commentaire supplémentaire.

La question qui se pose concernant cet item est celle de l'existence des arguments implicites. C'est un fait notoire que chaque verbe sélectionne un certain nombre de compléments qui sont plus ou moins nécessaires à définir son sémantisme (TESNIÈRE 1959). Les propriétés lexicales des verbes déterminant les caractéristiques d'une proposition,on peut distinguer plusieurs types d'arguments: (a) ceux qui ne peuvent en aucun cas être omis (p.e. «il a barré » qui est sans un argument, comme par exemple, « la route »); (b) ceux qui ont un statut flottant et peuvent disparaître sans que cela change le type d'événement représenté (p.e. "j'ai mangé »); (c) ceux qui sont déjà englobés dans le verbe (p.e. «j'ai téléphoné » où l'instrument utilisé, en l'occurrence le téléphone, demeure sous-entendu). À propos de (a) et (b), LAZARD (1994: 70) précise qu'il existe une catégorie intermédiaire, le type « habiter», qui demande l'explicitation d'un argument (et donc * «j'habite») dont toutefois la forme est variable, p.e. «j'habite Paris, j'habite à Paris, j'habite en France, etc.» (contrairement à «barrer», dont la construction ne prévoit jamais de préposition). Suivant l'hypothèse de JEŽEK (2018), un mécanisme d'effacement, que l'auteure appelle "defaulting », est responsable de l'omission des arguments. Un tel mécanisme s'active soit au niveau pragmatique (car l'information manquante est inférée à partir du co-texte ou du contexte, comme par exemple dans « hier j'ai commencé à $8 \mathrm{~h} 30$ ») soit au niveau lexical (car très souvent l'argument est absent comme dans le cas de «manger» ou « lire »). Dans la tradition française, l'attention est moins sur le processus que sur le statut de l'objet. On parle plus volontiers d'objet latent (dont le référent est à chercher dans le discours qui précèdeou bien dans leréservoir des connaissances encyclopédiques) ou bien d'objet non spécifique ${ }^{5}$, dit aussi générique, là où la référence n'est pas identifiable puisqu'il s'agit d'un procès habituel si bien que l'objet n'est plus pertinent, comme dans le cas de "que fait-il? il enseigne" (LARJAVAARA 2019: 39-60). Les deux approches, toutefois, ne sont pas incompatibles. L'emploi non spécifique correspond au « defaulting » lexical, car l'argument non exprimé se réfère à une

5 Suivant la terminologie très claire de WINAND (2004). 
classe générique d'entités, p.e. «il a mangé à $8 \mathrm{~h}$ », qui présuppose n'importe quel élément de la classe des choses que l'on peut manger. L'emploi spécifique correspond au "defaulting " pragmatique, car il s'agit d'une omission d'un élément déterminé d'une classe, p.e. "as-tu pris le médicament ? oui j'ai pris », qui sous-entend un élément précis de la classe des choses que l'on peut prendre. Dans ces deux typologies des omissions argumentaleson n'indique pas comment il faut ranger de tels emplois en fonction de la notion de marque, s'ils sont proches ou lointains d'un modèle prototypique. Il semblerait tout de même qu'on les considère plutôt comme des structures marquées. Larjavaara parle de " cas particuliers », alors quel'argumentation de Ježek laisse présupposer que la forme canonique soit celle où tous les arguments sont explicités. À ce propos, on pourrait invoquer est l'«ambitransitivité » telle qu'elle est définie par CREISSELS (2006: 2), pour qui: « une proportion plus ou moins importante des verbes d'une langue peuvent figurer également en construction transitive ou en construction intransitive sans cesser de représenter le même type d'évènement " soit celle, plus radicale, de ROBERGE (2007: 148) pour qui: «ce qu'il y a de prototypique dans la catégorie $\mathrm{V}$ c'est justement la possibilité d'apparaître avec un complément »; ce qui signifie qu'une position d'objet direct est toujours présente au niveau syntaxique, la transitivité étant une propriété du prédicat plutôt que du contenu lexical du verbe.

Or, au moins depuis Bréal, une autre façon de concevoir les choses est défendable (BERNARD 1991). Fidèle à sa conception suivant laquelle la syntaxe libre précède historiquemet la syntaxe liée (les mots étant d'abord autonomes avant de développer des connexions stables), BRÉAL (1897) a été probablement un des premiers à parler d'une force transitive neutre, voire intransitive, qui expliquerait aussi bien la construction à objet zéro que la conception à objet générique. Qui plus est, la neutralité serait à la base des autres combinaisons de type transitif, car: « [...] les mots ont été créés pour avoir une pleine signification par eux-mêmes, et non pour servir à une syntaxe [...] » (BREAL 1897: 210). Une telle conception, que Bréal applique à la diachronie des langues, où finalement il a existé « [...] un état premier, pendant lequel a régné l'intransitivité seule » (BLINKENBERG 1969: 13), pourrait être étendue à la synchronie représentée par notre corpus en envisageant l'emploi générique comme le moins marqué. On ne considère pas une telle valeur prototypique de manière absolue et donc panchronique, mais spécifique à notre corpus. On sait d'ailleurs que la marque peut varier suivant les différentes situations communicatives (p.e. diatopiques et diastratiques) ${ }^{6}$. Or, rappelons-nous que dans le cas de « la fille caressait » un

\footnotetext{
${ }^{6}$ Sur la possibilité toujours présente que ce qui est marqué dans un certain contexte ne le soit pas forcément dans un autre, ce qu'on appelle généralement "l'inversion de marque », voir, par exemple, AGOSTINIANI (1988) et CIANCAGLINI (1994).
} 
tiers des participants accepte la construction intransitive (qui, dans ce cas précis, serait à ranger dans le type inergatif). Il semblerait donc que pour ces adolescents, pour qui cette phrase est tout à fait grammaticale la notion de « rection » n'est pas encore opérationelle et que l'autonomie du mot demeure le principe qui régit la phrase. Les commentaires sont sur ces points très éclairants qui ne manifestent pas l'idée d'un objet obligatoire qui serait récupérable par le contexte7. L'événement est plutôt envisagé dans sa généricité sémantique (p.e. « oui, elle peut le faire; c'est une personne; c'est un être humain; la fille fait une action; il y a un sens ») et moins dans sa possibilité syntaxique (p.e. " pas besoin d'un complément; oui, même si l'objet n'est pas précisé; on peut rajouter quelque chose; elle est moins complétée »). Au total, les justifications ne sont pas très nombreuses; cela s'explique bien par le fait qu'il s'agit tout de même d'un commentaire à une réponse positive, c'est-à-dire d'une acceptation complète de la phase qui généralement ne déclenche que très peu de justifications métalinguistiques. Les collégiens qui ont devéloppé une intuition négative, en revanche, sont plus nombreux. Il est intéressant de noter que la plupart de leurs réflexions mettent en évidence un problème de construction souvent avec un métalangage assez adéquat: «il manque le complément, elle caresse quoi?, il manque le nom, il faut un COD ».

\section{voiture »}

\section{Réponses métalinguistiques concernant « le papa éternuait la}

Si le linguiste n'éprouve aucune difficulté à prendre en considération une sensibilité grammaticale qui s'écarte de l'usage, car la linguistique est avant tout descriptive et non normative, que doit-il faire, en revanche, face à un comportement linguistique apparemment normal mais qui dévoile, après un examen plus détaillé, une représentation assez inattendue?

L'un des atouts de l'épreuve d'acceptabilité du THAM-2 est justement de permettre une évaluation simultanée et du niveau superficiel du « noticing » et de l'« understanding » métalinguistique qui ne sont finalement que les deux pôles du même continuum allant de la détection de l'anomalie jusqu'à sa correction à travers l'explicitation des connaissances linguistiques (SCHMIDT 1994 et 2001). Les tentatives actuelles d'amélioration de la structure du test visent à mieux préciser si l'utilisation d'une terminologie grammaticale adéquate est une condition incontournable pour qu'une justification soit considérée comme métalinguistique à part entière. Le protocole prévoit deux valeurs numériques, 1 et 2 , suivant les différentes capacités à expliquer les incongruités, la valeur 0 n'étant

\footnotetext{
${ }^{7}$ Dans un seul cas, un élève de 5ème eprime un jugement incertain en disant que la phrase serait
} acceptable si le « chat » était déjà dans le texte. 
finalement réservée qu'aux réponses absentes ou à celles oùl'on répète ce qui a été déjà remarqué dans la partie épilinguistique (réponses tautologiques) ${ }^{8}$. La question débattue concerne l'évaluation qualitative des degrés de conscience métalinguistique et de leur relation avec l'utilisation ou pas d'un métalangage approprié (WOLL 2019: 58), surtout lorsque l'intérêt n'est pas de type correctif (objectif typique dans un milieu scolaire) mais plutôt descriptif dans lequel, au contraire, on se contente d'observer des usages et des représentations sans y ajouter un cadre normatif. Or, concernant la partie métalinguistique de l'item « le papa éternuait », -dont, rappelons-le brièvement, les réponses épilinguistiques portant sur la détection de l'incongruité sont massivement conformes aux attentes (voir tab. 1, 2 et 3), à savoir négatives - il est intéressant de noter que la majorité écrasante des réponses montrent que les collégiens ont un bon niveau de réflexion tout en justifiant demanièrenon conformepar rapport à ce que le test prévoit comme contenu explicite recevable (grosso modo, on s'attend à une réponse qui mette en exergue l'anomalie formelle, engendrée par la présence d'un objet après un verbe à construction intransitive). Un deuxième aspect critique s'ajoute donc à celui que WOLL (2019) a déjà montré car le problème n'est plus seulement celui de la forme dans laquelle l'argumentation est exprimée mais aussi celui du contenu de cette réflexion. En fait, la plupart des collégiens ont argumenté en faisant référence à leurs connaissances encyclopédiques, l'incompatibilité syntaxique n'intervenant que dans de rares cas, ce qui, dans le protocole, devrait correspondre à une valeur 0 :

Table 4. Pourcentage de collégiens qui ont répondu ou pas à la question métalinguistique.

\begin{tabular}{|c|c|c|}
\hline classes & $\begin{array}{c}\text { présence d'une réponse } \\
\text { métalinguistique }\end{array}$ & $\begin{array}{c}\text { absence de réponse } \\
\text { métalinguistique }\end{array}$ \\
\hline 6ème & $53 \%$ & $47 \%$ \\
\hline 5 ème & $91 \%$ & $9 \%$ \\
\hline 4 ème & $96 \%$ & $4 \%$ \\
\hline
\end{tabular}

Table 5. Pourcentage de réponses de type enciclopédique ou syntaxique.

\begin{tabular}{|c|c|c|}
\hline classes & $\begin{array}{c}\text { réponse de type } \\
\text { encyclopédique }\end{array}$ & réponse de type syntaxique \\
\hline 6ème & $87 \%$ & $13 \%$ \\
\hline 5ème & $88 \%$ & $12 \%$ \\
\hline 4ème & $90 \%$ & $10 \%$ \\
\hline
\end{tabular}

8 Pour la partie épilinguistique, les valeurs numériques sont 0 et 1 (réponse correcte/réponse incorrecte) alors que dans l'évaluation de la conscience métalinguistique, le test prévoit trois nineaux: 0 (absence d'argumentation), 1 (argumentation partiellement développée), 2 (argumentation complète). 
DEUX GROUPES DE COLLÉGIENS (ABIDJAN ET TOULOUSE) FACE À DEUX QUESTIONS SUR LA TRANSITIVITÉ ...

Par encyclopédique on indique une réponse où l'élève, puisqu'il ne prend en considération ni le fait que " éternuer » est un verbe à construction intransitive (ce qui impliquerait une réflexion sur la syntaxe) ni la possibilité qu'il s'agisse d'un problème d'interprétation (ce qui impliquerait une réflexion sur l'énonciation), s'appuie avant tout sur ses connaissances du monde qui l'entoure. C'est donc une justification axée davantage sur des aspects sémantiques et pragmatiques oùle sujet évalue le degré de plausibilité entre ce qui est dit et ce qui est possibile pour lui dans le monde objectif. La plupart du temps, les collégiens expliquent que l'événement est « impossibile », qu'il " n’a pas de sens » car « on ne peut pas faire sortir une voiture, qui est un objet lourd, des narines d'un homme». C'est une évidence tirée de leur expérience et non un raisonnement sur la bonne formation ou l'interprétabilité de la phrase. Les rares commentaires plus centrés sur la forme, en revanche, pointent soit l'absence d'une préposition «sur » ou « dans » qui permettraient de rétablir la cohérence de la phrase soit le fait qu'on n'a pas affaire à un verbe comme «laver» ou " essuyer», ce qui semble impliquer que la notion d'intransitivité est opérationnelle bien qu'exprimée de manière assez informelle.

Ce qui semble remarquable est le fait que le THAM-2 permette de sonder en profondeur la qualité du refus épilinguistique à « le papa éternuait la voiture » en dévoilant qu'il n'a pas un fondement syntaxique mais plutôt sémanticopragmatique. Compte tenu de l'âge, ce comportement est très intéressant. À nos yeux, il ne témoigne ni de ce que DE MAURO (1970: 93) appelle la linguistique du "sens commun », héritière de la conception aristotélicienne, suivant laquelle le langage est une "peinture» de la pensée, une nomenclature donc, ni d'un glissement du jugement d'acceptabilité grammaticale de la phrase vers une appréciation sur l'interprétation contextuelle de l'énoncé. Il montre plutôtque le besoin de donner du sens prime sur le respect de l'agencement formel.

\section{Discussion et conclusions}

La psycholinguistique et la linguistique sont généralement considérées comme des disciplines très proches, mais qui visent à des objectifs différents. $\mathrm{Si}$ la première s'intéresse aux opérations mentales, la deuxième analyse les produits de ces opérations et tente de les systématiser. Comme nous le rappelle un heureux manuel qui, unique en son genre, propose une exposition synoptique de ces deux approches pour chaque niveau d'analyse (LAUDANNA et VOGHERA 2006), le couple «structure/processus » peut nous guider à identifier la frontière labile qui délimite ces deux disciplines. Le linguiste s'intéresse davantage à la description des éléments (phonèmes, morphèmes, lexiques, syntagmes, etc.) et à leur cohérence dans une théorie qui les 
interprète. Le psycholinguiste étudie les élaborations mentales de ces « objets » linguistiques, c'est-à-dire les mécanismes cognitifs, en production et réception, qui sont à leur base. Cette contribution voudrait revenir sur le débat concernant l'utilité d'une intégration entre les deux champs disciplinairesen montrant que le THAM-2, par la façon dont ilest conçu, apporte des données très intéressantes qui méritent une attention particulière.

Premièrement, il y a un aspect théorique. La notion d'acceptabilité demande un approfondissement supplémentaire, pas uniquement de type sociologique (genre, âge, lieu, classe sociale, etc.). En effetil peut y avoir un écart assez important d'ordre psychologique entre le jugement polaire (oui/non) qui apparaît en surface et les raisons plus profondes qui pourraient le déterminer. Par conséquent, il n'est pas seulement important de distinguer la grammaticalité de l'énonçabilité (DESCLÉS et GUENTCHÉVA 1991) mais aussi d'essayer de définir, autant que faire se peut, sur quelles connaissances et sur quels types de raisonnement s'appuie un tel jugement. C'est ce qui émerge de manière évidente dans le cas de « le père éternuait la voiture ». Les collégiens ont rejetécette phrase de manière presque unanime mais en n'etayant pas leurs discoursavec des raisons formelles, comme on aurait pu le croire compte tenu de leur âge et de leur parcours scolaire. Dans le cas de «la fille caressait», en plus, il est intéressant de soulignerun deuxième aspect. Les raisons du « oui » ne sont pas symétriques à celles des "non», les premières étant plutôt de nature sémantico-pragmatique, les deuxièmes plutôt syntaxiques. De tout cela il émerge que si l'on réduit la recherche à la seule prise en compte du niveau épilinguistique, qui est certainement un point de départ nécessaire, on risque d'oublier la pensée qui se cache derrière une telle surface et qui est pourtant assez riche et variable. Sans le complément apporté par l'examen, parfois assez difficile, du niveau métalinguistique, le phénomène linguistique risque d'être envisagéseulement comme un comportement objectif et semi-automatique, sans plus, ce qui oblige à travailler uniquement sur des formes sans faire intervenir la notion plus problématique de « conscience linguistique ».

On pourrait rapprocher ce type de problème à celui que l'on rencontre dans l'analyse du comportement politique.On sait que dans l'adhésion au même projet politique (qui s'exprime notamment à travers le vote, lors des élections) les personnes sont sous l'emprise de multiples influences, parfois contradictoires entre elles: tradition de famille, origine géographique et sociale, effet de prestige d'un leader politique, saillance d'un thème spécifique du programme, calcul stratégique suivant ses propres intérêts, apriori idéologiques, etc. Deux électeurs du même parti, donc, peuvent être "guidés "par des motivations parfois très différentes tout comme les collégiens qui ont participé à notre enquête.

Comme on l'a peut-être remarqué, tout cela ouvre un champ de recherche autonome, qui vise à sonder plus les « intentions » et "l'intériorité » 
que les produits linguistiques ou les processus cognitifs. Reste à savoir quel est l'espace de légitimité d'un tel domaine de recherche appliquée à la langueet quelles pourraient être les méthodologies lesplus adéquates pour le décrire. Pour le moment, nous observons que le THAM-2, grâce à la possibilité d'observer en même temps des aspects du processus et des produits mais surtout grâce au fait qu'il donne la parole aux sujets qui « agissent » dans leur langue ouvre l'accès à une subjectivité en évitant de la traiter comme un objet externe, comme le ferait le discours scientifique avec son métalangage 9 .

Deuxièmement, il y a un aspect empirique concernant la question de l'âge. Nos données semblent montrer que bien au-delà des huit ans, on peut encore ne pas savoir séparer le contenu d'une phrase de sa forme, du moins dans des situations où il n'est pas demandé explicitement de le faire. Dans le THAM-2, en effet, on se limite à poser des questions polaires sur l'acceptabilité puis à demander d'argumenter les réponses sans orienter davantage le comportement des sujets, si ce n'est que par le fait que chaque input anomal, p.e. " le père éternuait la voiture », est précédé d'un item correct, «le père éternuait »contenant la même séquence à un détail près, ce qui aurait pu favoriser des réactions s'appuyant sur une analyse syntaxique.

Nous avançons l'hypothèse que dans l'interprétation d'un item la première étape de l'étudiant est de vérifier la compatibilité entre l'apparat conceptuel et la structure sémantique, c'est-à-dire la correspondance d'abord entre le type sémantique et le rôle thématique des arguments (en l'occurrence « la fille /personne, agent/, " " père /personne, agent/ », " voiture /artéfact, patient/ ") avec le sémantisme des verbes. Seulement ensuite entrent en jeu les rôles syntaxiques et la valence. Ces premiers résultats, surtout lorsqu'on considèrequ'il s'agit toujours des mêmes collégiens qui sont plus ou moins convergents dans leurs réponses et non pas de deux groupes différents, laissent supposer que cette « approche sémantique » qui se différencie de l'« approche formelle » est moins une étape cognitive qu'une stratégie toujours disponible à laquelle on pourrait recourir de manière différente en fonction non seulement de la maturité due à l'âge mais aussi des connaissances explicites acquises. Les données concernant « la fille caressait » d'ailleurs montrent bien que l'analyse basée sur des raisons linguistiques commence à devenir plus importante, du moins dans certains cas, ce qui pourrait indiquer qu'une transformation est en cours, chez nos collégiens. Cette deuxième hypothèse aussi, qui est plus conforme à une optique vygotskyenne du développement linguistique où desnouvelles opérations mentales peuvent émerger, les plus anciennes se

\footnotetext{
${ }^{9}$ Dans le domaine des sciences humaines, pour une défense d'une méthodologie qualitative qui valorise l'intériorité comme elle se produit et ne la traite pas de l'extérieur, tel un objet, ce qui correspond typiquement à la posture dite « scientifique », voir (LAZARUS: 1996).
} 
transformer, sans jamais disparaître définitivement ${ }^{10}$, mériteune réflexion théorique. Elle pourrait être vérifiée, par exemple, avec d'autres enquêtes sur les intuitions des étudiants universitaires comme celle de LACHET (2015) ou bien en administrant aux adultes le THAM-2 dans les mêmes conditions des nos collégiens.

\section{BIBLIOGRAPHIE}

AGOSTINIANI, Luciano, « Marcatezza, lingue funzionali e fenomeni di ristrutturazione del parlato in Toscana », in: ALBRECHT, Jörn, LÜDTKE, Jens, THUN, Harald (éds), Energeia und Ergon. Sprachliche Variation-Sprachgeschichte-Sprachtypologie. Studia in honorem Eugenio Coseriu, Tübingen, Narr, 1988, p. 441-455.

BEACCO, Jean-Claude, "Les savoirs linguistiques "ordinaires" en didactique des langues: des idiotismes », Langue française, 2001, p. 89-105.

BERNARD, Gilles, «Une conception linguistique méconnue de la transitivité », Linx, 1991, p. 13-35.

BLINKENBERG, Andreas, Le problème de la transitivité en français moderne, København, Munskgaard, 1969.

BRÉAL, Michel, Essai de sémantique, Paris, Hachette, 1897.

BRÉDART, Serge, RONDAL Jean-Adolphe, L'analyse du langage chez l'enfant: les activités métalinguistiques, Sprimont, Mardaga, 1982.

CIANCAGLINI, Angela, « Per una valutazione dei fondamenti teorici della marcatezza », in: CIPRIANO, Palmira, DI GIOVINE, Paolo, MANCINI, Marco (éds), Miscellanea di studi in onore di W. Belardi, II tomo, Roma, Il Calamo, 1994, 811-845.

COLOMBO, Adriano, «La riflessione grammaticale: riflessioni di un conservatore », in: AMBEL, Mario (éd), Insegnare la lingua. Quale grammatica? Milano, Edizioni scolastiche Bruno Mondadori, 1982. p. 12-70.

CREISSELS, Denis, Syntaxe générale, une introduction typologique, 2, la phrase, Paris, Lavoisier.

CRYSTAL, David, A Dictionary of Linguistics and Phonetics, Oxford, Blackwell Publishing, 2008.

DEBRENNE, Michèle, «Les représentations métalinguistiques des étudiants en FLE », in: HILTON, Heater E. (éd.), Acquisition et didactique1, Chambéry, Université de Savoie, 2007, p. 55-66.

DE MAURO, Tullio, Introduzione alla semantica, Bari, Laterza, 1970.

${ }^{10}$ Sur cet aspect de l'idée du développement chez Vygotsky, héritage d'une vision hégelo-marxiste de l'histoire comme rapport instable entre tradition et innovation et non comme une séquence linéaire d'étapes, voir, par exemple, le débat avec Piaget qui croit en la disparition du langage égocentrique chez l'enfantalors que Vygostky soutient qu'il se transforme en langage intérieur (VYGOTSKY 1954: 36). 
DEUX GROUPES DE COLLÉGIENS (ABIDJAN ET TOULOUSE) FACE À DEUX QUESTIONS SUR LA TRANSITIVITÉ ...

DESCLÉS, Jean-Pierre, GUENTCHÉVA Zlatka, «Test et acceptabilité », Histoire Épistémologie Langage, 1991, p. 9-25.

DE VILLIERS, Peter A., DE VILLIERS, Jill G., « Early Judgments of Semantic and Syntactic Acceptability by Children », Journal of Psycholinguistic Research, 1972, p.299-310.

GOMBERT, Jean Émile, Le développement métalinguistique, Paris, Puf, 1990.

JEŽEK, Elisabetta, "Partecipanti impliciti nella struttura argomentale dei verbi », in: DALLABRIDA, Sara, CORDIN, Patrizia (éds), La grammatica delle valenze. Spunti teorici, strumenti e applicazioni, Firenze, Franco Cesati Editore, 2018, p. 55-71.

LACHET, Caroline, «Pratiques et représentations grammaticales des étudiants à l'université », in: KALMBACH, Jean-Michel, STRATILAKI-KLEIN, Sofia (éds), Descriptions linguistiques et descriptions pédagogiques pour l'enseignement et l'apprentissage du français, Actes du 2 e colloque international du GRAC, en ligne, 2015, p. 70-81.

LA MANTIA, Francesco, «"Un atteggiamento irenico". Su alcune pagine culioliane di Tullio De Mauro » Bollettino del centro di Studi Filologici e Linguistici Siciliani, 2017, p. 151-174.

LARJAVAARA, Meri, La transitivité verbale en français, Paris, Ophrys, 2019.

LAUDANNA, Alessandro, VOGHERA, Miriam, Il linguaggio. Strutture linguistiche e processi cognitivi, Roma-Bari, Laterza, 2006.

LAZARD, Gilbert, L'actance, Paris, Puf, 1994.

LAZARUS, Sylvain, Anthropologie du nom, Paris, Seuil, 1996.

NADEAU, Marie, FISHER Carole, « Les connaissances implicites et explicites en grammaire: quelle importance pour l'enseignement ? Quelles conséquences ?», Bellaterra Journal of Teaching and Learning \& Literature, 2011, p. 1-31.

PINTO, Maria Antonietta, La consapevolezza metalinguistica. Teoria, sviluppo e strumenti di misurazione, Pisa-Roma, Istituti editoriali e poligrafici internazionali, 1999.

PINTO, Maria Antonietta, EL EUCH, Sonia, La conscience métalinguistique, Québec, PUL, 2015.

PETERFALVI, Jean-Michel, LOCATELLI, Françoise, "L'acceptabilité des phrases », L'année psychologique, 1971, p. 417- 427.

ROBERGE, Yves, «Structure de transitivité verbale en français », in: BOUCHARD, Denis (éd.), Représentation du sens linguistique II. Actes du colloque international de Montréal (2003). Louvain-la-Neuve, De Boeck Supérieur, 2007, p. 137-150.

SCHMIDT, Richard, " Deconstructing consciousness in search of useful definitions for applied linguistics », AILA Review, 1994, p.11-26.

SCHMIDT, Richard, "Attention», in: ROBINSON, Peter (éd), Cognition and second language instruction, Cambridge, Cambridge university Press, 2001, p. 3- 32.

SORACE, Antonella, « Metalinguistic knowledge and language use in acquisition-poor environments », Applied Linguistics, 1985, p. 239-254.

TESNIÈRE, Lucien, Éléments de syntaxe structurale, Paris, Klincksieck, 1959.

TITONE, Renzo, Psicolinguistica applicata, Roma, Armando Armando, 1983.

TRÉVISE, Anne, « Représentations métalinguistiques des apprenants, des enseignants et des linguistes: un défi pour la didactique », Bulletin VALS-ASLA, 1994, p. 171-190. 
TUNMER, William E., GRIEVE Robert, Syntactic awareness in children, in: TUNMER, William E., PRATT, Chrisopher, HERRIMAN Michael L. (éds), Metalinguistic Awareness in Children: Theory, Research, and Implications, Berlin, Heidelberg, New York, Tokyo, Springer-Verlag, 1984, p. 92-104.

VYGOTSKY, Lev S., Pensiero e linguaggio, Firenze, Giunti-Barbèra, 1954.

WEBER, Corinne, «La culture grammaticale ordinaire: étude de verbalisations métagrammaticales et métacognitives d'apprenants natifs ». Langages, 2004, p. 101-112.

WEBER, Corinne, "Les verbalisations ordinaires dans la classe: objets furtifs ou variables encombrantes des sciences du langage ?», Pratiques, 2008, p.139-140.

WINAND, Jean, «La non-expression de l'objet direct en égyptien ancien», Lingua Aegyptia, 2004, p. 205-234.

WOLL, Nina, «How French speakers reflect on their language: a critical look at the concept of metalinguistic awareness », Language Awareness, 2019, p. 49-73. 